\title{
Caracterización Morfológica y Agronómica de la Colección Nacional de Germoplasma de Jatropha curcas $L$.*
}

\section{Morphological and Agronomic Characterisation of the Jatropha curcas L. Colombian Germplasm collection}

\author{
Jorge A. Guerrero Pinilla1*, Luis F. Campuzano ${ }^{2 \star}$, Salvador Rojas ${ }^{3 *}$ \\ Jorge Pachon-García ${ }^{*}$ \\ ${ }^{1}$ Ingeniero Agrónomo, Universidad de los Llanos \\ 2,3Ingeniero Agrónomos PhD, Corpoica \\ ${ }^{4}$ Biólogo MSc. Universidad de los Llanos \\ *jotapachon@hotmail.com
}

Recibido: Agosto 23 de 2011. Aceptado: Noviembre 8 de 2011

\section{RESUMEN}

Con el objetivo de estimar la diversidad genética de la colección colombiana de germoplasma de Jatropha curcas L., con un total de 246 accesiones colectadas en estado silvestre en distintas regiones del país, se llevó a cabo una caracterización morfológica y agronómica mediante la medición de 27 características genéticas de tipo cualitativo y cuantitativo. El estudio se basó en métodos de estadística multivariada para hallar la variabilidad genética entre las accesiones. Las distancias obtenidas en el dendograma posibilitaron formar 18 grupos al estimar el coeficiente de disimilaridad DICE entre los caracteres asociados a componentes de producción, características agronómicas y descriptores de interés específico, asociados a 10 componentes principales que logran explicar el 78,25\% de la variación. La caracterización morfológica permitió determinar la variabilidad genética dentro de las accesiones en especial los descriptores de disposición de arquitectura de planta, porte de las plantas, longitud de la parte de la rama no lignificada y color del látex. La importancia del estudio radica en el manejo y uso de estas accesiones en programas de mejoramiento genético tradicional o asistido por marcadores moleculares conforme al aprovechamiento de la especie en la agroindustria con fines energéticos en la producción de agrocombustibles.

Palabras clave: Descriptores, Variabilidad Genética, Jatropha curcas L., Agrocombustibles. 


\section{ABSTRACT}

In order to determine the genetic diversity of the colombian germplasm collection of Jatropha curcas L., established with a total of 246 wild accessions collected in different regions of the country, it was carried out a morphological and agronomical characterization using 27 genetic traits either qualitative or quantitative type. The study was based on multivariable statistics statistical to determine the genetic variability among accessions. The distances in the dendrogram made possible to stablish 18 groups based on the DICE dissimilarity coefficient between the characters associated with yield components, agronomic characteristics, and specific descriptors of interest related to 10 principal components obtained from 20 quantitative evaluated descriptors. Morphological characterization allowed us to determine the presence of genetic variability within accessions especially descriptors available to plant architecture, plant size, length of the not lignified branch, latex color and this study of the Jatropha germplasm collection allows the handling and use of accessions in traditional plant and molecular markers assisted programs according to the use of the species for energy purposes in the production of agrofuels.

Key words: Descriptors, Genetic Variability, Jatropha curcas L., Agrofuels.

\section{INTRODUCCIÓN}

El uso de especies de uso agrícola en las últimas décadas para la producción de agrocombustibles ha generado una búsqueda intensa para identificar nuevos recursos fitogenéticos con un alto potencial en la producción de biocombustibles sin poner en riesgo la seguridad alimentaria de los países. El problema se ha convertido no solo en un asunto de carácter agroindustrial sino que ha tomado una dimensión ética al ocasionar una disminución en la oferta de alimentos para favorecer la producción de biodiesel o bioetanol. En este contexto, el caso de el piñón de purga, Jatropha curcas L., usado popularmente para fines medicinales (Adolf et al., 1984), surge como una excelente alternativa no solo porque su composición de ácidos grasos lo hace ideal para producción de un biodiesel de notable calidad sino porque además, su uso podría disminuir la presión que se produce sobre la demanda de oleaginosas liberándolas para la alimentación humana y animal.

Jatropha curcas L., es una especie perenne de la familia Euforbiaceae, que no ha sido caracterizada completamente y el conocimiento de sus características fenotípicas y genotípicas es limitado aunque se han documentado hasta el momento ciertas características botánicas. $J$. curcas es un arbusto de crecimiento rápido que alcanza en promedio seis metros de altura, su corteza es blanco-grisácea y exuda un látex translúcido color ámbar o rojizo viscoso. Los tallos crecen de manera simpodial con una discontinuidad morfológica en cada incremento. Normalmente se forman cinco raíces de cada arbusto, una central y cuatro periféricas (Toral, 2008). Las hojas se forman generalmente con cinco a siete lóbulos acuminados pocos profundos y grandes, tienen pecíolos largos con una longitud de diez a quince centímetros; anchura de nueve a quince centímetro, de forma ovada, las cuales caen durante la época seca (Guerrero, 2010).

Las inflorescencias se forman terminalmente en el axial de las hojas en las ramas, compuestas a manera de panícula, en la cual, el eje principal se ramifica una o más veces y puede sostener las umbelas. En flores hermafroditas se colocan diez estambres en dos espirales distintas de cinco cada uno en una sola columna en el androceo. En el gineceo, tres estilos delgados son conatos en aproximadamente dos tercios de su longitud, dilatando al estigma bifurcado macizo. Ambas flores, masculinas y femeninas, son pequeñas (6$8 \mathrm{~mm}$ ), verdoso-amarillas en su diámetro y pubescente. Los frutos son cápsulas drupáceas y ovoides. Después de la polinización se forma una fruta trilocular de forma elipsoidal. Las frutas son cápsulas inicialmente verdes que se tornan café 
oscuro o negro en el proceso de maduración. Las cápsulas de los frutos son de 2.5 a $4 \mathrm{~cm}$ de largo por $2 \mathrm{~cm}$ de ancho, elipsoidales y lisas que al inicio son carnosas pero dehiscentes cuando son secas (Mishra, 2009). Cada inflorescencia rinde un manojo de aproximadamente 10 frutos ovoides o más. El desarrollo del fruto necesita 90 días desde la floración hasta que madura la semilla (Heller, 1996). La fruta produce tres almendras negras por lo general, cada una aproximadamente de $2 \mathrm{~cm}$ de largo y $1 \mathrm{~cm}$ de diámetro.

La semilla se cosecha cuando la cápsula está madura. La maduración se evidencia cuando esta cambia de verde a amarillo lo cual ocurre después de dos a cuatro meses de la polinización. Las semillas se usan comúnmente como laxante al tiempo que las evidencias actuales indican que estas presentan una proporción de aceite del $48 \%$ (Panigrahi et al., 1984).

La especie presenta actualmente limitantes de manejo en cultivo para fines industriales por su bajo nivel de domesticación. Se cree que la estructura genética de sus poblaciones es bastante heterogénea debido a que su asincronía floral (Downes y Wold, 1994). favorece el proceso de polinización cruzada. En J. curcas se presenta una gran divergencia durante el desarrollo de las fases masculina y femenina en la inflorescencia ya que se libera primero el polen a medida que se manifiesta el crecimiento de los estilos y los estigmas en el gineceo con una menor precocidad en el desarrollo de las papilas estigmáticas Debido a esta

\section{MATERIALES Y MÉTODOS}

\section{Colección de germoplasma}

Entre el año 2007 y 2008, se colectaron 246 accesiones de Jatropha curcas L., en diversas zonas del país (Tabla 1). La colección se encuentra establecida en un lote de 2.5 ha. del Centro de Investigación "La Libertad" de La Corporación asincronía en la floración dentro de la inflorescencia, se origina gran una desuniformidad en los ciclos de producción y esto se convierte en una característica que se debe mejorar a la hora de hablar de esta especie como un posible cultivo de explotación comercial (Henning, 2004).

La necesidad de una caracterización primaria y secundaria se justifica entonces no solo por el conocimiento que se genera en la fenología de la especie sino que para propósito de mejoramiento genético es indispensable conocer la biología reproductiva y estructura genética de la especie. De esta manera, se podrá conocer el nivel de variación genética que existe dentro de especie y el potencial que presentan algunos de los materiales de la colección de germoplasma para que sean incorporados en un programa de mejoramiento genético. En ese orden de ideas vale la pena destacar los avances realizados por Brasil (Laviola et al., 2011), la India (Gohil y Pandya, 2008) y Perú (Manco y Pérez, 2009). Colombia por su parte, ha iniciado sus esfuerzos con una caracterización morfofisiológica realizada por la Universidad Nacional de material proveniente del Brasil en el cual se evaluaron siete descriptores cualitativos en las ecoregiones de Vichada y Santander (Pedraza y Cayón, 2010).

El objetivo del presente estudio fue determinar la diversidad genética de la colección de germoplasma de Jatropha curcas $L$. del centro de investigaciones "La Libertad" de la Corporación Colombiana de Investigación Agropecuaria, CORPOICA.
Colombiana de Investigación Agropecuaria CORPOICA, Villavicencio. Cada accesión cuenta con 12 plantas. Para los efectos de este trabajo se muestrearon aleatoriamente 3 plantas por accesión. 
Tabla 1. Resumen de colecta de accesiones

\begin{tabular}{cc}
\hline ZONA DE & $\begin{array}{c}\text { NÚMERO TOTAL DE } \\
\text { COLECTA }\end{array}$ \\
\hline Casanare & 33 \\
Caquetá & 1 \\
Guaviare & 18 \\
Meta & 114 \\
Vichada & 35 \\
Atlántico & 8 \\
Bolívar & 9 \\
Cesar & 11 \\
Guajira & 13 \\
Córdoba & 9 \\
Sucre & 5 \\
Antioquia & 7 \\
Boyacá & 2 \\
Caldas & 2 \\
Cundinamarca & 2 \\
Huila & 1 \\
Tolima & 35 \\
\hline TOTAL & $\mathbf{3 0 5}$ \\
\hline Fuente: Corpoica, 2009 & \\
&
\end{tabular}

Fuente: Corpoica, 2009

\section{Localización}

La caracterización se llevó a cabo en el Centro de Investigaciones "La Libertad", ubicado en Villavicencio sobre la vía a Puerto López a una latitud de $4^{\circ} 03^{\prime} 40,25^{\prime \prime} \mathrm{N}$ y longitud $73^{\circ} 26^{\prime} 57,82 " \mathrm{~W}$. Este sitio se caracteriza por presentar una alta pluviosidad (3000 a 4000 mm/año), humedad relativa superior al $85 \%$, alta radiación solar, y oxisoles con altos contenidos de arcilla y una saturación de aluminio superior al $70 \%$.

\section{Caracterización morfológica y evaluación}

La tabla 2 muestra los diferentes caracteres usados para la caracterización morfológica. Estos incluyeron tanto características cualitativas como cuantitativas. Las medidas se realizaron sobre el tercio medio de la planta para evitar escapes según los descrito por González y Fita (2001). Se seleccionaron 20 características de tipo cuantitativo y 7 cualitativos para un total de 27 posibles descriptores (Campuzano, 2009) agrupados en componentes de producción, características agronómicas y descriptores de interés específico (EMBRAPA, 2007).

Las particularidades y categorías de algunos descriptores que a continuación se describen se encuentran en la tabla 3. La altura de las plantas se determinó a los 12 meses después de germinadas las semillas y se establecieron cuatro categorías: Porte bajo $(<95 \mathrm{~cm})$, medio (95-170 $\mathrm{cm})$, alto $(170-260 \mathrm{~cm})$, muy alto (>260). Con respecto a la tendencia del hábito de crecimiento de las plantas hay que tener en cuenta que debido al crecimiento simpodial las ramas laterales se desarrollan más que el eje principal. Para este descriptor se tuvo en cuenta la particularidad del crecimiento, ya sea en Monocasio o Dicasio. 
Tabla 2. Descriptores botánicos propuestos para Jatropha (Jatropha curcas L.)

\begin{tabular}{|c|c|c|}
\hline $\begin{array}{l}\text { DESCRIPTORES } \\
\text { PROPUESTOS }\end{array}$ & \multicolumn{2}{|c|}{ TIPO DE CARACTERÍSTICA } \\
\hline $\begin{array}{l}\text { COMPONENTES DE } \\
\text { RENDIMIENTO }\end{array}$ & CUANTITATIVA & CUALITATIVA \\
\hline Número de Ramas /planta & * & \\
\hline Número de Racimos / Rama & $*$ & \\
\hline Número de Frutos / Racimo & $*$ & \\
\hline Número de semillas / Fruto & $*$ & \\
\hline Peso de las Semillas & * & \\
\hline Porte de la Planta & & $*$ \\
\hline $\begin{array}{l}\text { Tendencia del hábito de } \\
\text { crecimiento simpodial }\end{array}$ & & * \\
\hline $\begin{array}{l}\text { Longitud de rama foliar no } \\
\text { lignificada }\end{array}$ & & * \\
\hline $\begin{array}{l}\text { Disposición de arquitectura en la } \\
\text { planta }\end{array}$ & & $*$ \\
\hline Ángulo de bifurcación & & * \\
\hline Color del Látex & & * \\
\hline \multicolumn{3}{|l|}{$\begin{array}{l}\text { CARÁCTERISTICAS } \\
\text { AGRONÓMICAS }\end{array}$} \\
\hline Altura de Plantas & * & \\
\hline Diámetro del Tallo & $*$ & \\
\hline Proyección de la Copa & $*$ & \\
\hline Longitud de la Hoja & $*$ & \\
\hline Anchura de la Hoja & $*$ & \\
\hline $\begin{array}{l}\text { Razón longitud / Anchura de la } \\
\text { Hoja }\end{array}$ & $*$ & \\
\hline Días a floración & * & \\
\hline Peso de Frutos & * & \\
\hline Longitud de Frutos & $*$ & \\
\hline Anchura de Frutos & $*$ & \\
\hline $\begin{array}{l}\text { Razón Longitud / Anchura de } \\
\text { Frutos }\end{array}$ & $*$ & \\
\hline Longitud de las Semillas & * & \\
\hline Anchura de las semillas & $*$ & \\
\hline $\begin{array}{l}\text { Razón Longitud / Anchura de las } \\
\text { Semillas }\end{array}$ & * & \\
\hline \multicolumn{3}{|l|}{$\begin{array}{l}\text { DESCRIPTORES DE } \\
\text { INTERÉS ESPECÍFICO }\end{array}$} \\
\hline Uniformidad de Maduración & & $*$ \\
\hline
\end{tabular}

Para la longitud de rama foliar no lignificada, se midieron dos ramas diferentes de la misma planta desde el ápice hasta el punto en que la rama cambia su textura herbácea blanda a una textura más rígida y lignificada (Tabla 3) se establecieron tres categorías: Larga ( $>50 \mathrm{~cm}$ ), Mediana ( 25 y $50 \mathrm{~cm}$ ) o Corta $(<25$ $\mathrm{cm})$. En la disposición de arquitectura de la planta se determinó si la tendencia del crecimiento era de tipo ortotrópico (vertical) o plagiotrópico. El ángulo de bifurcación se estableció como el ángulo con que se desarrollan las ramas de la planta con respecto al eje principal del cual provienen y se asignó como recto $\left(90^{\circ}\right)$, agudo $\left(15^{\circ}-90^{\circ}\right)$ u obtuso $\left(>90^{\circ} \mathrm{y}<\right.$ $\left.180^{\circ}\right)$.

El látex de Jatropha contiene en promedio un $10 \%$ de pigmentos taninos (Heller 1996) y se clasificó de acuerdo con su apariencia como rojo o blanco ámbar. 
La unidad de maduración de los racimos se estableció como el porcentaje de frutos maduros y se midió como alta ( $>75 \%)$, media ( $75-25 \%)$ y baja (<25\%).

Los datos se organizaron en una matriz para facilitar el almacenamiento, acceso y recuperación de los mismos. El análisis estadístico de la información se realizó mediante el programa INFOSTAT 2010. Los caracteres evaluados se evaluaron a través de los métodos de componentes principales y de correspondencia múltiple. Estos métodos se fundamentan en la reducción de la dimensionalidad de la estructura original de los datos de tal manera que se facilita la visualización de las interrelaciones entre accesiones y entre descriptores. El análisis multivariado permitió establecer los caracteres con mayor capacidad discriminatoria y aquellos que lograronn explicar la mayor parte de la variación. Se realizó un análisis de correlación entre todas las variables cuantitativas con la finalidad de detectar variables altamente correlacionadas y se establecieron los clusters que agrupaban a las accesiones con mayores similitudes.

Con la matriz de datos establecida se generó un dendograma a través de la técnica de agrupamiento aglomerativo de Ward, empleada comúnmente para conocer correlacionados entre grupos de poblaciones.

Tabla 3. Porcentaje de incidencia interespecífica de caracteres cualitativos

\begin{tabular}{|c|c|c|c|}
\hline \multirow{3}{*}{ DESCRIPTOR } & \multicolumn{3}{|c|}{ PORCENTAJE DE } \\
\hline & CUALIDAD & INCIDENCIA & FRECUENCIA \\
\hline & & INTERESPECÍFICA \% & \\
\hline \multirow{4}{*}{ Porte de la Planta } & Bajo $(<95 \mathrm{~cm})$ & 1,72 & 4 \\
\hline & Medio $(95-170 \mathrm{~cm})$ & 14,22 & 33 \\
\hline & Alto $(170-260 \mathrm{~cm})$ & 71,98 & 167 \\
\hline & Muy alto $(>260)$ & 12,08 & 28 \\
\hline \multirow{2}{*}{$\begin{array}{l}\text { Tendencia del hábito de } \\
\text { crecimiento simpodial }\end{array}$} & Dicasio & 72,8 & 169 \\
\hline & Monocasio & 27,2 & 63 \\
\hline \multirow{3}{*}{$\begin{array}{l}\text { Longitud de rama foliar no } \\
\text { lignificada }\end{array}$} & $(\operatorname{Larga}(>50 \mathrm{~cm})$ & 5,17 & 12 \\
\hline & Mediana $(25$ y $50 \mathrm{~cm})$ & 13,8 & 32 \\
\hline & Corta $(<25 \mathrm{~cm})$ & 81,03 & 188 \\
\hline \multirow{2}{*}{$\begin{array}{l}\text { Disposición de } \\
\text { arquitectura en la planta }\end{array}$} & Plagiotrópica & 40,0 & 93 \\
\hline & Ortotrópica & 60,0 & 139 \\
\hline \multirow{3}{*}{ Ángulo de bifurcación } & Recto $\left(90^{\circ}\right)$ & 18,1 & 42 \\
\hline & Agudo $\left(15^{\circ}-90^{\circ}\right)$ & 50,4 & 117 \\
\hline & Obtuso $\left(>90^{\circ} \mathrm{y}<180^{\circ}\right)$ & 31,5 & 73 \\
\hline \multirow{2}{*}{ Color del Látex } & Rojo & 0,43 & 1 \\
\hline & Blanco Ám bar & 99,57 & 231 \\
\hline Uniformidad de & Alta $(>75 \%)$ & 30,6 & 71 \\
\hline $\begin{array}{l}\text { Maduración / Racimo } \\
\text { (porcentaje de frutos }\end{array}$ & Media $(75-25 \%)$ & 65,95 & 153 \\
\hline maduros) & Baja $(<25 \%)$ & 3,45 & 8 \\
\hline
\end{tabular}




\section{RESULTADOS Y DISCUSIÓN}

\section{Base de datos}

La base de datos pertenece al grupo de investigación en J. curcas liderado por CORPOICA. Esta base contiene toda la información de las accesiones en cuanto a los datos de pasaporte y, los datos de la caracterización morfológica y evaluación agronómica.

\section{Caracteres cualitativos}

Los resultados de la caracterización de los caracteres cualitativos se encuentran reportados en la tabla 3. Los descriptores cualitativos propuestos fueron aplicados a cada carácter heredable previamente reportado, expresado morfológicamente por las plantas durante las observaciones en campo. El hábito de crecimiento de las plantas, el desarrollo vegetativo, la posición de la inflorescencia, los rasgos particulares de la semilla en cada accesión, la longitud de rama foliar no lignificada; la disposición de arquitectura en la planta (Plagiotrópica - Ortotrópica); Ángulo de bifurcación; Color del Látex (Rojo - Blanco Ámbar); y la Uniformidad de Maduración / Racimo mostraron variaciones que permiten suponer que existen la suficiente variación genética dentro de la colección para establecer un programa de mejoramiento de dichas características.

\section{Caracteres cuantitativos}

Los resultados permitieron agrupar las 232 accesiones entre 18 grupos diferentes (tabla 8). Los valores propios de la matriz de correlaciones permiten establecer que 10 de los 20 descriptores aplicados logran explicar el $78.25 \%$ de la variación (Tabla 4).

Tabla 4. Valores propios de la matriz de correlación

\begin{tabular}{|c|c|c|c|c|}
\hline \multicolumn{5}{|c|}{ VALORES PROPIOS DE LA MATRIZ DE CORRELACIÓN } \\
\hline & Valor propio & Diferencia & Proporción & Acumulado \\
\hline 1 & 2,99440352 & 0,76055 & 0,1497 & 0,1497 \\
\hline 2 & 2,23385348 & 0,2391375 & 0,1117 & 0,2614 \\
\hline 3 & 1,99471601 & 0,2137604 & 0,0997 & 0,3611 \\
\hline 4 & 1,78095563 & 0,4085034 & 0,089 & 0,4502 \\
\hline 5 & 1,37245228 & 0,1705454 & 0,0686 & 0,5188 \\
\hline 6 & 1,20190685 & 0,0856365 & 0,0601 & 0,5789 \\
\hline 7 & 1,11627038 & 0,004971 & 0,0558 & 0,6347 \\
\hline 8 & 1,11129935 & 0,1763765 & 0,0556 & 0,6903 \\
\hline 9 & 0,93492284 & 0,0260102 & 0,0467 & 0,737 \\
\hline 10 & 0,90891264 & 0,0627099 & 0,0454 & 0,7825 \\
\hline 11 & 0,84620279 & 0,0706241 & 0,0423 & 0,8248 \\
\hline 12 & 0,77557866 & 0,0265562 & 0,0388 & 0,8636 \\
\hline 13 & 0,74902251 & 0,0453815 & 0,0375 & 0,901 \\
\hline 14 & 0,70364103 & 0,2028513 & 0,0352 & 0,9362 \\
\hline 15 & 0,50078977 & 0,1006928 & 0,025 & 0,9612 \\
\hline 16 & 0,40009695 & 0,0993405 & 0,02 & 0,9813 \\
\hline 17 & 0,30075643 & 0,2323001 & 0,015 & 0,9963 \\
\hline 18 & 0,06845636 & 0,0633482 & 0,0034 & 0,9997 \\
\hline 19 & 0,00510816 & 0,0044538 & 0,0003 & 1,000 \\
\hline 20 & 0,00065438 & & 0,0000 & 1,000 \\
\hline
\end{tabular}


La identificación de estos caracteres permite entonces reducir el número de descriptores con alto valor discriminatorio para caracterizar en el futuro los materiales con alto un alto potencial para la producción de biodiesel. De acuerdo con sus valores propios, las características de mayor interés son: la longitud de la semilla, el ancho de la semilla, la relación largo y ancho de la semilla, número de semillas por fruto, peso de las semillas, número de frutos por racimo, número de ramas por planta, Enfocarse en estas caracteristicas permitirá reducir los costos a la hora de caracterizar los materiales.

Al organizar las accesiones en grupos según el poder discriminatorio de los caracteres heredables, se aplicaron estadísticos simples como la varianza y la relación de medias con el fin de conocer el rango de variación superior o inferior de los datos con respecto a la media aritmética para cada variable cuantitativa (Tabla5).

Tabla 5. Estadísticos simples aplicados en variables cuantitativas a todas las accesiones

\begin{tabular}{|c|c|c|c|c|}
\hline VARIABLES CUANTITATIVAS & MEDIA & $\begin{array}{l}\text { DESVIACIÓN } \\
\text { ESTANDAR }\end{array}$ & $\begin{array}{l}\text { LÍMITE } \\
\text { MÁXIMO }\end{array}$ & $\begin{array}{l}\text { LÍMITE } \\
\text { MÍNIMO }\end{array}$ \\
\hline Altura de Planta $(\mathrm{cm})$ & 205,225 & 49,762 & 99 & 344 \\
\hline Diámetro del Tallo (cm) & 7,157 & 1,199 & 44 & 111 \\
\hline Proyección de la Copa $(\mathrm{cm})$ & 176,395 & 57,436 & 71 & 287 \\
\hline Longitud de la 8a Hoja (cm) & 13,900 & 2,548 & 8,6 & 18 \\
\hline Ancho de la 8a Hoja (cm) & 13,131 & 2,33 & 9,6 & 18 \\
\hline Relación Largo /Ancho de la hoja & 1,083 & 0,253 & 0,7 & 1,5 \\
\hline Días a Flora ción & 359,807 & 30,663 & 299,7 & 454 \\
\hline Peso del Fruto (g) & 7,828 & 2,066 & 4,6 & 13 \\
\hline Longitud del Fruto $(\mathrm{cm})$ & 3,002 & 0,361 & 2,4 & 3,5 \\
\hline Ancho del Fruto $(\mathrm{cm})$ & 2,754 & 0,441 & 1,6 & 3,4 \\
\hline Relación Largo / Ancho del Fruto & 1,105 & 0,139 & 0,9 & 2 \\
\hline Longitud de la Semilla (cm) & 1,795 & 0,045 & 1,4 & 1,8 \\
\hline Ancho de la Semilla (cm) & 0,900 & 0,007 & 0,8 & 0,9 \\
\hline Relación Largo / Ancho de la Semilla & 1,995 & 0,054 & 1,6 & 2,1 \\
\hline Uniformidad de Maduración (\%) & 66,708 & 16,574 & 20,8 & 90,66 \\
\hline $\mathrm{N}^{\circ}$ Ramas / Planta & 3,446 & 0,807 & 1,6 & 5,8 \\
\hline $\mathrm{N}^{\circ}$ Racimos / Rama & 2,016 & 0,605 & 0,6 & 3 \\
\hline $\mathrm{N}^{\circ}$ Frutos / Racimo & 4,468 & 1,758 & 1,2 & 8 \\
\hline $\mathrm{N}^{\circ}$ Semillas / Fruto & 2,989 & 0,061 & 2 & 3 \\
\hline Peso Semillas (g) & 1,243 & 0,128 & 0,6 & 1,5 \\
\hline
\end{tabular}


Dentro de cada grupo en que se han clasificado las diferentes accesiones, se hizo una jerarquización según la cercanía de caracteres fenotípicos correlacionados entre sí, con lo cual se pueden estimar de manera comparativa en cada uno de los 18 grupos las accesiones con caracteres fenotípicos más incipientes, con base en la clasificación y selección de atributos obtenidos por conglomerados de Ward. Se encontró que el grupo de accesiones con mayor altura de plantas es el grupo 11, procedentes de Sucre, Córdoba, Cesar, Región Andina y Meta; cuyo promedio de altura es de 2,76 $\mathrm{m}$, dentro de un rango que oscila entre 2,35 y $3,16 \mathrm{~m}$ a los 18 meses de edad (Tabla 6). En contraste con otros estudios realizados en condiciones del trópico húmedo de Brasil, se han encontrado grupos de accesiones en que la mayor altura es de $1.87 \mathrm{~m}$ al año después del trasplante (Laviola, et. al, 2011), en India, de los cinco clúster en que se agruparon las accesiones según sus características más afines la mayor altura se expresa en materiales con 1,53 m en plantas de un año después del trasplante, por lo tanto, hay que tener en cuenta que el porte de las plantas provenientes de la zona norte de Colombia son de mayor porte en comparación a las demás regiones del país e incluso las de India.

El mayor diámetro de tallo se observó entre las accesiones seleccionadas en el grupo 7, procedentes de zonas como la Guajira, Atlántico, Córdoba, Bolívar y sucre, excepto algunas procedentes de Vichada y Casanare con $84,5 \mathrm{~mm}$, en promedio dentro de un rango de $78,4 \mathrm{~mm}$ y $90,7 \mathrm{~mm}$ a los 18 meses (Tabla 6). Dicho atributo en otras regiones como India presentan $13,57 \mathrm{~cm}$ de diámetro al año después del trasplante. La proyección de la copa con mayor cobertura la poseen las accesiones del grupo 12, provenientes de zonas del país como Córdoba, Sucre, Meta, Vichada y Antioquia, en las cuales el promedio fue $244 \mathrm{~cm}$, dentro de un rango de variación que va desde $200 \mathrm{~cm}$ hasta $287 \mathrm{~cm}$ (Tabla 6). Con esta característica el contraste de los materiales de una y otra región dentro de Colombia presentan una mayor incidencia de crecimiento Plagiotrópico, muy semejante a lo reportado por los materiales caracterizados en Brasil donde la cobertura de la copa en plantas de un año alcanzan $197,5 \mathrm{~cm}$, mientras que en India presentan una mayor proyección de hasta $410 \mathrm{~cm}$ a los 16 meses luego del trasplante, siendo de vital importancia para designar distancias de siembra. En dicho grupo 12 la octava hoja presenta las mayores dimensiones con respecto a las demás accesiones, cuyo promedio de longitud oscila entre $15,22 \mathrm{~cm}$ y $17,88 \mathrm{~cm}$, con $16,55 \mathrm{~cm}$ en promedio, mientras el ancho se estima en $16,68 \mathrm{~cm}$, dentro de un rango de $16,09 \mathrm{~cm}$ y $17,27 \mathrm{~cm}$. Por lo tanto la relación largo / ancho tiende a ser la más uniforme de los grupos obtenidos, cuyo índice es de 1,0 con una variación que va de 0,93 hasta $1,07 \mathrm{~cm}$.

En cuanto a los días a floración las accesiones con mayor precocidad pertenecen al grupo 3, procedentes de Sucre, Meta, Guaviare, Casanare y Antioquia, las cuales en promedio a los 333 días contados desde su siembra en vivero empiezan a florecer después de germinar la semilla, dicho dato puede variar pues algunas emergieron entre 300 y 366 días (Tabla 6). Las accesiones menos precoces corresponden al grupo 1, provenientes de Antioquia y Meta, las cuales florecen 464 días después de germinar la semilla y su rango de variación está entre 438 y 490 días. Este dato contrasta con los materiales de menor precocidad reportados por Laviola en Brasil, de los cuales la juvenilidad se estima en 206 días en promedio contados después del trasplante, debido a la arquitectura de dichos materiales y la distribución de las yemas florales en mayor número por rama, pero con menor tamaño de frutos y semillas con respecto a las accesiones colombianas.

El mayor peso de fruto se obtuvo entre las accesiones asociadas al grupo 9, procedentes de Cesar, Casanare, Vichada y Meta, con un peso promedio de cada fruto estimado en $11,97 \mathrm{~g}$, dentro de un rango de variación de 10,9 g a 13g (Tabla 6). En cuanto a las dimensiones del fruto la mayor longitud se presenta en el grupo 9 con 3,27 cm en promedio, dentro de un rango de 3,1 a $3,4 \mathrm{~cm}$. El ancho de cada fruto en promedio es mayor en el grupo $3 \mathrm{con} 3,15 \mathrm{~cm}$, oscilando entre $3 \mathrm{y}$ $3,3 \mathrm{~cm}$. La mayor uniformidad de los frutos los presenta el grupo 8, compuesto por accesiones provenientes de Meta, Vichada, Casanare y Centroamérica, con un índice de uniformidad de 1,01, con una leve variación que se mantiene en el rango de 0,9 y 1,06 (Tabla 6). La longitud de la semilla en las accesiones del grupo 14 es mayor con respecto a los demás 
Tabla 6. Procedencia de las accesiones de cada grupo con características significativas

\begin{tabular}{|c|c|c|c|c|c|c|}
\hline \multicolumn{7}{|c|}{ PROCEDENCIA DE LOS GRUYOS CON CARACTERS TICAS MÁS S IGNIFICATTVAS } \\
\hline GRY0 & $\frac{\text { ACCESION }}{3.6171819 \eta+6,055960.168 .}$ & \multirow{2}{*}{$\begin{array}{l}\text { PROCEDENCIA } \\
\text { META } \\
\text { ANTTO OUIA }\end{array}$} & \multirow{2}{*}{ CARACTERISTICA } & \multicolumn{3}{|c|}{ ?R0MEDIOGMTE MÁXOMIMITE MINM } \\
\hline 1 & $\begin{array}{l}3,6,17,18,19,7,46,4,56,59,60,168 \\
138,137\end{array}$ & & & 4643 & 438,4 & 490,2 \\
\hline \multirow{5}{*}{3} & 142 & REGTÓ N ANDINA & & & & \\
\hline & $126,191,192,106,198$ & VICHADA & < DLAS A FLORACIÓN & 333,1 & 299,7 & 366,4 \\
\hline & 99 & GTAMLARE & & & & \\
\hline & $\begin{array}{l}75 \\
86,94121,127,140,170,183 .\end{array}$ & $\begin{array}{l}\text { SUCRE } \\
\text { META }\end{array}$ & & & & 3275 \\
\hline & $97,167,199$ & CASANARE & 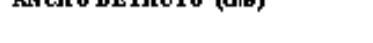 & 3,1516 & 3,0201 & 0,2100 \\
\hline \multirow{5}{*}{4} & 8198 & GJAVLARE & & & & \\
\hline & $\begin{array}{l}76 \\
10.25 .28 .38 .40 .44 .56 .66 .69 .77 .86 .\end{array}$ & $\begin{array}{l}\text { BOLFAR } \\
\text { META }\end{array}$ & NÚMERO DE FRUT/RACM O & 6,9 & 6,3 & 7,4 \\
\hline & $67: 217$ & $\begin{array}{l}\text { CASANARE } \\
\text { CESAR }\end{array}$ & & & & \\
\hline & $\begin{array}{l}70,74 \\
88,202,209,212\end{array}$ & $\begin{array}{l}\text { REGTON ANDINA } \\
\text { VICHADA }\end{array}$ & FES O DESEMIILA (gnS) & 1,2839 & 1,265 & 1,3063 \\
\hline & 208 & SUCRE & & & & \\
\hline \multirow{6}{*}{5} & $\begin{array}{l}29.53 .54 .64 .107 .108 .116 .135 .155 .165 .23 .225 \text {. } \\
145,109\end{array}$ & $\begin{array}{l}\text { META } \\
\text { CASANARE }\end{array}$ & & & & \\
\hline & 125 & GTALARE & & & & \\
\hline & 139 & SUCRE & & 8501 & 8107 & 8896 \\
\hline & $\begin{array}{l}146 \\
15 ?\end{array}$ & $\begin{array}{l}\text { GNAIRA Y ATLANTI } \\
\text { CESAR }\end{array}$ & $\mathrm{C}^{\text {UNIF OBMIDAD DEMADURACION }}$ & 85,01 & 8,07 & 00,90 \\
\hline & $\begin{array}{l}157 \\
158,159,179,26\end{array}$ & $\begin{array}{l}\text { CESAR } \\
\text { VICHADA }\end{array}$ & & & & \\
\hline & 180 & ANTTO OUIA & & & & \\
\hline \multirow{7}{*}{7} & 106 & GTAJRA Y ATLANTI & & & & \\
\hline & 110.113 & SUCRE & & & & \\
\hline & 123,187 & BOLIVAR & & & & \\
\hline & 129 & META & DLAMETR0 DE TALLO (tIm) & 84,54 & 78,42 & 90,66 \\
\hline & $\begin{array}{l}148 \\
149,177,181\end{array}$ & $\begin{array}{l}\text { GJAVIARE } \\
\text { CASANARE }\end{array}$ & & 64,54 & 70,42 & 90,00 \\
\hline & 163 & CORDOBA & & & & \\
\hline & $175: 188$ & VICHADA & & & & \\
\hline \multirow{4}{*}{8} & $23,26,30,31,32,33,35,39,41,49,51,65,71,73,80$ & META & & & & \\
\hline & $72: 102: 114$ & CASANARE & LARGO /ANCHO DE FRUTO & 1.01 & 0.96 & 106 \\
\hline & 87 & EXTRANTERAS & & & & \\
\hline & 174 & VICHADA & NÚMERO DE RACM ORAMA & 2,52 & 2,28 & 2,75 \\
\hline \multirow{5}{*}{9} & $105: 213$ & META & & & & \\
\hline & 131 & CESAR & & & & \\
\hline & $134,141,150,161,162$ & CASANARE & LONGTUD DE FRUTO (ans) & 3,274 & 3,128 & 3,42 \\
\hline & $151: 152,214915$ & VICHADA & & & & \\
\hline & 176 & BOLIVAR & & & & \\
\hline \multirow{8}{*}{11} & 62 & REGTO N ANDINA & & & & \\
\hline & $63: 185: 216$ & CORDOBA & & & & \\
\hline & $85.102: 115,173$ & META & & & & \\
\hline & 120 & CESAR & ALTURR DEPLANTA (GOS) & 2764 & 35.5 & 315.8 \\
\hline & 122 & CASANARE & ALIUKA DEYLANTA (ans) & 270,04 & & \\
\hline & 124 & GTAJRA Y ATLANTI & & & & \\
\hline & 172 & VICHADA & & & & \\
\hline & 210 & SUCRE & & & & \\
\hline \multirow{7}{*}{12} & 6122 & CORDOBA & FROYECCIÓN DE COPA (ans) & 24,75 & $200: 44$ & 287,06 \\
\hline & 93 & VICHADA & LONGTTUD DE HOJA (ans) & 16,55 & 15,22 & 17,88 \\
\hline & 128,203 & META & & & & \\
\hline & 193 & ANTTO OUIA & ANCH O DE HOJA (́NS) & 16.68 & 16.09 & 17.27 \\
\hline & 201 & CORDOBA & & & & \\
\hline & $m 1$ & SUCRE & LARGO /ANCHO DE H OJA & 1.001 & 0.931 & 1.072 \\
\hline & 52,119 & META & & & & \\
\hline \multirow{3}{*}{13} & 164 & GTAJIRA Y ATLÁNTI & $c_{\text {MUJURO DE RaMASTLANTA }}$ & 46 & 39 & 53 \\
\hline & 171 & VICHADA & 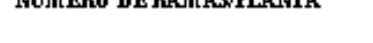 & & & \\
\hline & $15,43,45,143,166$ & $\begin{array}{l}\text { LASANAHE } \\
\text { META }\end{array}$ & & & & \\
\hline & 92 & GTAJTRA Y ATLÁATI & & & & \\
\hline 14 & 133 & REGTO N ANDINA & LONGTUD DESEMIILA (ans) & 1,8167 & $1,7 m 2$ & 1,850 \\
\hline & 242 & EXTRANDERAS & & & & \\
\hline & 27 & META & & & & \\
\hline 16 & 91 & GUANLARE & ANCF O DE SFMILLA (OTS) & 003 & * & 夫 \\
\hline 10 & 147 & REGTÓ N ANDINA & ANCHO DESEMILLA (OMS) & 0,93 & & \\
\hline & 241 & EXTRANDERAS & & & & \\
\hline 17 & 94.48 .57 .58 .101 .153 & META & LARGO /ANCHO DE SEMIILA & 1,72 & 1,59 & 1,85 \\
\hline
\end{tabular}


grupos, con un promedio de $1,82 \mathrm{~cm}$, encontrándose semillas con una longitud desde 1,78 hasta $1,86 \mathrm{~cm}$, la procedencia de dicho grupo es de la región Andina, Meta y Centroamérica. El ancho de la semilla es mayor en aquellas accesiones pertenecientes al grupo 16, procedentes de la región Andina, Meta y Centroamérica con 0,93 $\mathrm{cm}$ en promedio, sin presentar variación alguna. La dimensiones largo / ancho tienden a manifestarse de manera más uniforme en el grupo 17, provenientes del Meta con un índice de uniformidad de 1,72 , variando entre 1,59 y 1,85 . La uniformidad de maduración es mayor en las accesiones del grupo 5 con el $85 \%$ de uniformidad entre los frutos de cada racimo, oscilando entre $81,0 \%$ y $88,96 \%$, cuyas entradas proceden de Sucre, Antioquia, Meta, Vichada y Casanare. Este carácter en las accesiones caracterizadas en Colombia presenta un menor tamaño en relación con los reportados en las accesiones colectadas y establecidas en Perú (Manco y Pérez, et. al, 2009), los cuales llegan a medir $1,94 \mathrm{~cm}$ de largo y $1,18 \mathrm{~cm}$ de ancho, con un índice de uniformidad de 1,63 .

El mayor número de ramas por planta se encontró en las accesiones del grupo 13, procedente de Guajira, Atlántico, Meta, Casanare y Vichada, con 4,6 ramas en promedio oscilando entre 4 a 5 ramas por planta. El mayor racimo de frutos por rama se halló entre las accesiones del grupo 8, procedente de Casanare, Meta y Vichada en el cual el promedio fue de 3 racimos. En cuanto al número de frutos por racimo en el grupo 4, procedente de Casanare, Meta, Vichada y Guaviare se encontró el mayor promedio, el cual es de 7 frutos, con semillas que en promedio pesan $1,31 \mathrm{~g}$, dentro de un rango de $1,24 \mathrm{~g}$ y $1,38 \mathrm{~g}$.

En el estudio de las características morfológicas y agronómicas cuantitativas se presentan los promedios estimados a nivel general para todas las accesiones, con lo cual se encontró que las accesiones midieron en promedio $205,22 \mathrm{~cm}$ al aplicar el descriptor Altura de Planta (AP). Al comparar dicho promedio entre las accesiones colectadas en Colombia, aquellas que proceden de zonas como Córdoba, la Región Andina, Vichada y Casanare presentan los niveles máximos de crecimiento en altura, las cuales poseen un porte muy alto, superior a $260 \mathrm{~cm}$ de longitud, mientras que las plantas de las accesiones que se encuentran por debajo del promedio proceden con mayor incidencia de Centro América. El Diámetro de Tallo (DT), entre las distintas accesiones evaluadas presenta en promedio de $7,16 \mathrm{~cm}$ con una desviación estándar poco significativa de $\pm 1,2$ $\mathrm{cm}$, así mismo es evidente la gran diversidad de plantas con proyección de copa diferentes, que con respecto al promedio $(176,395 \mathrm{~cm})$, los datos colectados tienden a estar $\pm 57,436 \mathrm{~cm}$ respecto al promedio, lo cual es evidente al incidir en la tendencia del crecimiento simpodial ya sea dicotómico en Monocasio o en dicasio, debido a su arquitectura Plagiotrópica, para algunas accesiones y Ortotrópica para otras. En cuanto a los descriptores longitud y ancho de la octava hoja después de la inflorescencia, se encontraron promedios poco diferenciables para la gran mayoría de accesiones; con 13,9 cm, y 13,131 cm correspondientemente, con lo cual, se obtuvo un índice de relación entre el largo y ancho de la hoja de 1,1 , lo que permite tener una percepción de las hojas con tendencia a ser más largas que anchas, con un área laminar siempre dispuesta a capturar la presencia de luz gracias a su ubicación helicoidal siempre hacia el extremo de las ramas, haciéndose más escasa la presencia de hojas después de la octava hoja, ya que cuando las hojas alcanzan dicha posición en las ramas, las hojas presentan su mayor área láminar adquirida durante su crecimiento (Tabla 5).

Los días a floración de manera general en las accesiones evaluadas se estima en un periodo cercano al año, ya que en promedio las plantas florecen por primera vez 360 días después de germinar la semilla, con una desviación estándar que manifiesta la tendencia a seguir produciendo las primeras inflorescencias 31 días antes o después. El peso del fruto para todas las accesiones evaluadas se promedia en $7,828 \mathrm{~g}$, con $\pm 2,066 \mathrm{~g}$ de desviación, lo que indica la gran diversidad del peso del fruto entre todas las accesiones. Mientras que el índice de uniformidad del fruto, estimado mediante la relación largo y 
ancho, se promedia en 1,1 , lo cual indica la tendencia a encontrar entre las accesiones frutos más largos que anchos, los cuales, en promedio tienen $3,00 \mathrm{~cm}$ de longitud y $2,754 \mathrm{~cm}$ de ancho. El índice de uniformidad de las semillas se estima en promedio en 2 tendiendo a una forma elíptica con un promedio de $1,975 \mathrm{~cm}$ a lo largo y $0,9 \mathrm{~cm}$ de ancho, $\pm 0,054 \mathrm{~cm}$, esta es una característica poco variable entre las accesiones. La uniformidad de maduración es un descriptor cualitativo que se ha estimado como cuantitativo, al tener en cuenta la incidencia de frutos fisiológicamente maduros respecto a los frutos verdes en el mismo racimo, al ser evaluados en porcentajes de incidencia, de lo cual se encontró que en promedio el $66,72 \%$, de los frutos por racimo entre las accesiones tienden a estar maduros, mientras que el $33,29 \%$ de los frutos restantes, suelen encontrarse en estados fisiológicamente inmaduros. Dicho descriptor de uniformidad de maduración fue tomado tres meses después de contar el número de racimos de flores por rama, aplicado a plantas que presentaban inflorescencias en aquel momento entre 12 y 18 meses después de germinadas las semillas. Las 232 accesiones evaluadas poseen en promedio 3,5 ramas principales por planta según el descriptor número de ramas por planta, dicho dato varía $\pm 0,8$ ramas con respecto al promedio según la accesión evaluada. El número de racimos contados por rama, se estima en un promedio de 2,02 racimos en cada rama de las plantas, $\pm 0,6$ racimos respecto al promedio. De dichos racimos se ha estimado un promedio de 4,5 frutos bien formados por racimo, con tendencia a presentar \pm 2 frutos. El número de semillas por fruto, entre las accesiones es de 2,99 semillas por fruto, este dato puede variar en $\pm 0,06$ semillas, ya que la tendencia de cada planta a producir 3 semillas dentro de sus frutos es casi constante, pero puede presentarse una probabilidad muy pequeña de obtener frutos con dos semillas, e incluso cuatro, dichos caracteres aunque se han manifestado en los frutos colectados de algunas accesiones, no descartan la posibilidad de ser un fenómeno inducido por el ambiente de colecta.

En Colombia a partir de los 18 clusters jerarquizados, se acudió al diseño y elaboración de un dendograma, realizado con base en el cálculo de la media de todas las variables aplicadas a cada grupo o "cluster". Los clusters que se formaron fueron aquellos que obtuvieron el menor incremento en la suma de las distancias al cuadrado dentro de cada grupo. Teniendo en cuenta que la distancia euclídea al cuadrado depende de la medición de cada variable y el dato promedio obtenido en la aplicación de cada descriptor, con dicho cálculo se buscó estandarizar los datos con el fin de evitar que las variables tuvieran valores muy distintos a los reales en el cálculo de la distancia estimadas por el coeficiente de Nei y Li (Nei y Li, 1979). Se obtuvo el coeficiente de correlación cofenética, el cual permitió medir la correlación entre las interdistancias gráficadas en el dendograma, siguiendo las características fenotípicas de cada accesión dentro de una matriz de distancias Euclideas expresadas por el Coeficiente de Dice, las cuales evidencian que tan cercanos se encuentran los caracteres genéticos con respecto a la matriz de correlación, al presentarse mayor o menor variabilidad de estos entre las accesiones, lo cual contribuye a su expresión en el fenotipo valorado mediante los 20 descriptores cuantitativos (Figura 1). En contraste con otros estudios similares se reporta que en Brasil la caracterización de los 175 materiales estudiados, presentan una diferencia significativa del $5 \%$ de probabilidad entre accesiones para tomar las variables validadas, un coeficiente de variación de $72,3 \%$ y una heredabilidad del $27,16 \%$, menor a la estimada en la colección colombiana de CORPOICA.

\section{Matriz de correlación entre descriptores cuantitativos}

Se observó según el comportamiento de los datos, que existe un incremento directamente proporcional con respecto a la proyección de la copa, el diámetro de tallo, largo y ancho de la octava hoja y el peso del fruto con respecto a la altura de la planta. EI diámetro del tallo tiende a incrementarse de manera directamente proporcional según la altura de la planta, la proyección de la copa, el número de ramas por 
planta, el número de racimos por planta y la uniformidad de maduración. Con los resultados se observó la correlación inversa de los días a floración con respecto las alturas de las plantas. La proyección de la copa tiende a incrementarse proporcionalmente de a cuerdo a variables como la altura de la planta, el diámetro del tallo, la longitud y el ancho de la octava hoja, así mismo se ha encontrado directamente correlacionada con las dimensiones de los frutos, el peso de los frutos, el número de las semillas y el peso de las mismas. Sin embargo, se observa que los días a floración tienen una correlación inversa con respecto a la proyección de la copa. La longitud de la octava hoja, tiende a incrementarse de manera directamente proporcional con la altura de la planta, proyección de la copa, ancho de la octava hoja, el peso y las dimensiones del fruto. Al analizar los datos se observó entre los datos que al incrementar la medida del diámetro de los tallos en las accesiones la longitud de la octava hoja tiende a disminuir.

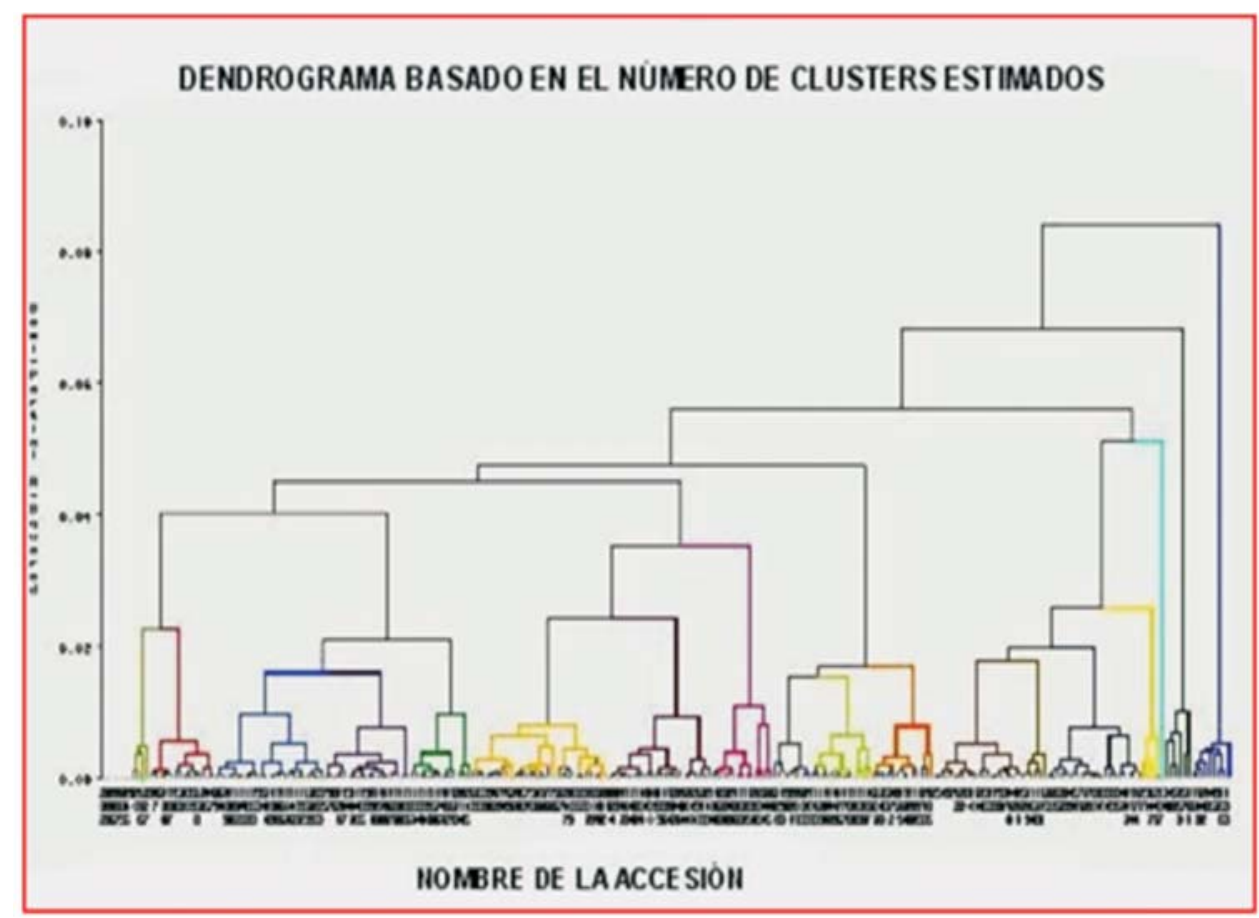

Figura 1. Grupos de accesiones jerarquizadas con base en el estudio de componentes principales al estimar las distancias Euclidianas, las cuales son explicadas con un $84 \%$ de disimilaridad entre el total de los grupos

El ancho de la octava hoja aumenta, con el ancho del fruto y los días a floración de manera generalizada entre todas las accesiones. No obstante la relación largo / ancho de la hoja se ve correlacionado directamente con características como dimensiones del fruto y número de frutos por racimo (Tabla 7).
Con respecto a los resultados de correlación del peso del fruto, se encontró que este dato se incrementa en la medida en que se presentan accesiones con plantas de mayor altura, mayor proyección de copa, longitud de la octava hoja y ancho del fruto. Por otro lado, la longitud del fruto presentaba datos cada vez mayores al encontrar accesiones donde tienden a incrementarse la proyección de la copa, la longitud de la octava hoja, el ancho del fruto y el peso de la semilla. 
Tabla 7. Matriz de correlación entre los descriptores cuantitativos aplicados

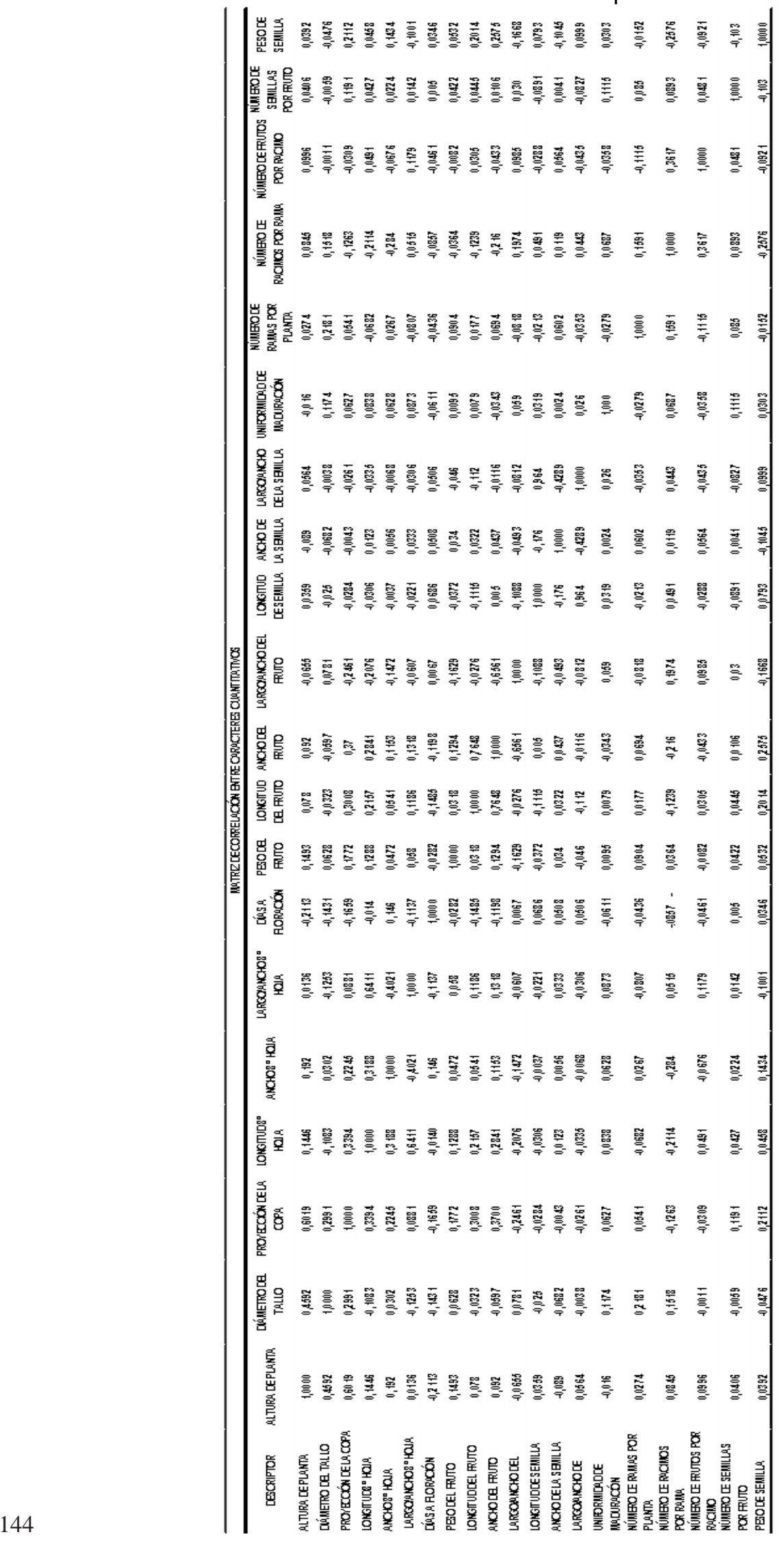


Tabla 8. Número total de accesiones agrupadas en cada clúster

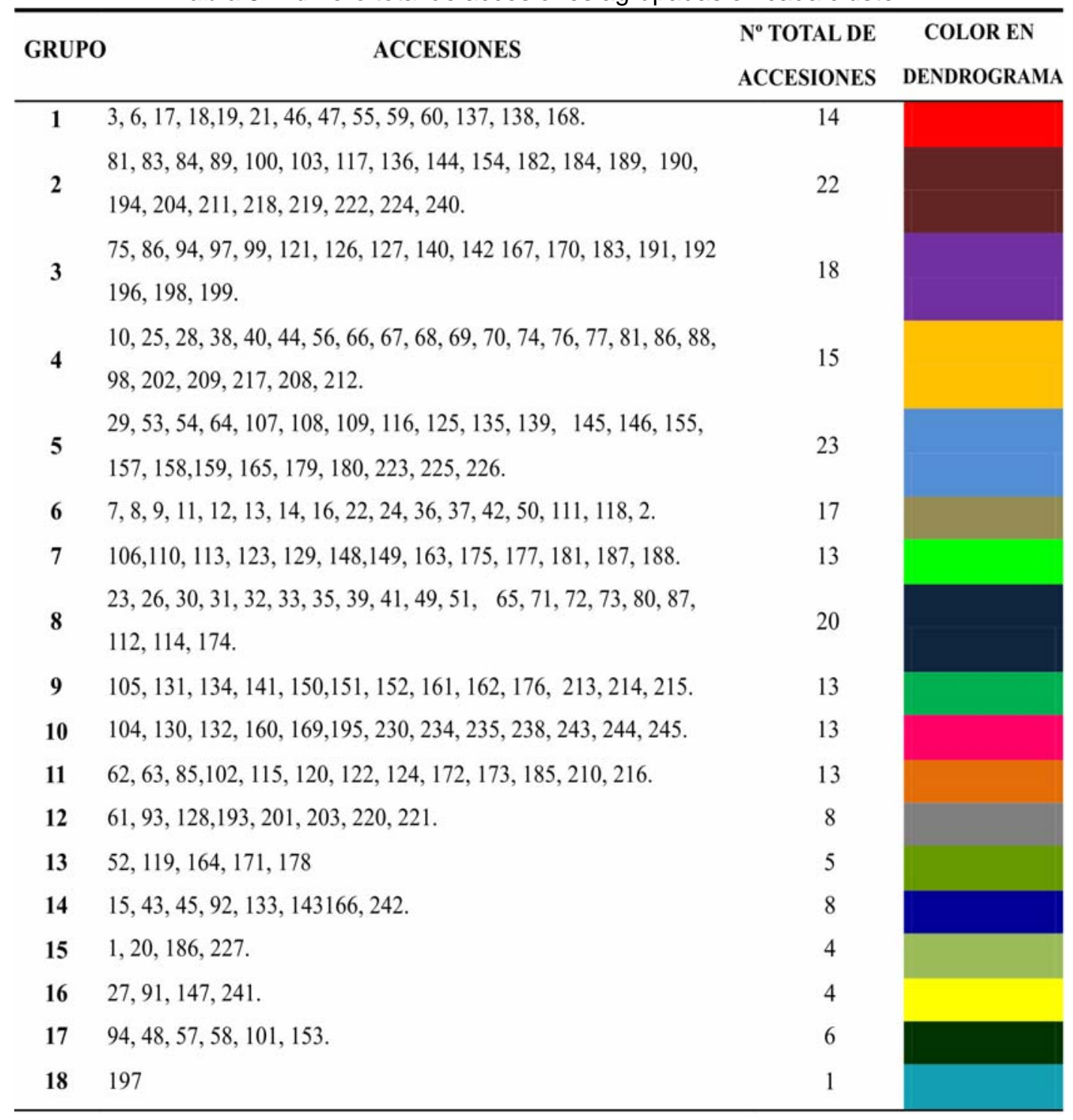

Los resultados obtenidos con respecto a la uniformidad de maduración revelan que al ser mayor el diámetro del tallo y número de semillas por fruto, la uniformidad de los frutos en cada racimo es mayor. Además, el número de frutos por racimo tiende a un aumento proporcional según el número de racimos por planta. La caracterización muestra que el peso de cada semilla tiende a aumentar de manera directamente proporcional el ancho y largo del fruto (Tabla 7).

Sumado a los resultados obtenidos, se proponen descriptores para estimar su poder discriminatorio al evaluar la incidencia de protandria entre accesiones como fenómeno reproductivo en la inflorescencia de Jatropha curcas L., debido a que las flores hermafroditas presentan asincronía floral durante el desarrollo de las fases masculina y femenina, ya que se libera primero el polen a medida que se manifiesta el crecimiento del Gineceo en los estilos y los estigmas, desarrollándose con menor precocidad las papilas estigmáticas. Se recomienda tomar los datos del conteo realizado al número de granos de polen adheridos, tanto al estilo como al estigma, el número de granos de polen germinados y el número 
de tubos polínicos que han aumentado su tamaño en longitud, haciéndose observable este fenómeno con el aumento de la longitud del estilo. No obstante, se ha observado que al valorar la incidencia de polinizadores incrementa la viabilidad reproductiva de la especie, sin embargo esto provoca una reducción en el número de flores que llegan a convertirse en frutos, ya que en promedio por cada 12 flores que se producen por umbela en el racimo se forman entre 6 y 8 frutos aproximadamente. Aunque el polen se conserva por un periodo corto en las anteras, una vez el androceo ha llegado a su etapa dehiscente los estigmas de la flor se hacen receptivos, presentando muy poca probabilidad de autopolinizaciones ya que la flor hermafrodita está iniciando la fase femenina (Bertín, 1993).

Las flores de Jatropha presentan 10 estambres, los cuales se hacen visibles con ayuda de una lupa, estos se hacen evidentes una vez la flor hermafrodita acaba de abrir, en un comienzo los estambres se elongan separando sus anteras mediante la dehiscencia longitudinal. En dicho momento el gineceo presenta

\section{CONCLUSIONES}

Se determinaron 18 grupos fenotípicos diferentes entre 232 accesiones para 20 características cuantitativas. Las características morfológicas con mayor poder discriminatorio fueron aquellas relacionadas con la disposición arquitectónica de la planta y mayor variabilidad en los grupos fenotípicos 2, 4, 5, 6, 7y 12 , lo que manifiesta la diversidad genética estimada con 24 descriptores con buen poder discriminatorio de los 26 aplicados durante la caracterización primaria (morfológica) y la evaluación caracterización secundaria. Al identificar los caracteres morfológicos con mayor poder discriminatorio para establecer diferencias entre las accesiones, se encontró que entre las características cuantitativas evaluadas, la mayor variabilidad para los caracteres con valores propios que explican el $78,25 \%$ de la variación genética para 10 caracteristicas en relación con 20 características propiamente morfológicas concernientes a los descriptores agronómicos, componentes de rendimiento y uniformidad de maduración como descriptor de interés específico; ya que la incidencia de caracteres dentro de las descriptores manifestaron polimorfismos expresados los estilos de un tamaño muy pequeño, lentamente los estilos van creciendo, mientras que el resto de los estambres maduran y liberan el polen. Una vez se ha producido la senescencia de las anteras, los estilos alcanzan su tamaño ideal y con las papilas ya desarrolladas. En dicho acto es necesario tomar una lupa para observar detalladamente la presencia de papilas ya desarrolladas. A lo largo del estilo se observan papilas a las cuales se les adhieren los granos de polen cuando ya están desarrolladas. Por lo tanto, es necesario conocer la longitud del estilo para analizar la longitud adecuada para la adhesión de los granos de polen y de igual manera conocer la presencia de tubos polínicos ya fecundados acudiendo al método de epifluorescencia (Kearns y Inouye, 1993). Con dichos descriptores se busca encontrar si existe realmente protandria en la accesión a estudiar, realizando el análisis previo a la receptividad del estigma, así como a la incidencia del polen en el estigma, y el periodo en que se hace posible la autogamia espontanea, aun conociendo que en la especie predomina la alogamia como método de multiplicación natural.

en el fenotipo de las plantas entre accesiones con sólo el $50 \%$ del total de las características evaluadas.

Para concluir las apreciaciones obtenidas acerca de las características expresadas en las flores de la planta de Piñón (Jatropha curcas L.), se ha manifestado divergencia durante el desarrollo de las fases masculina y femenina de cada inflorescencia en una misma cohorte, al realizar reiterativamente observaciones en campo y aplicar los descriptores concernientes a los componentes de rendimiento, ya que se libera primero el polen de las flores masculinas en la medida en que se manifiesta el crecimiento del Gineceo y la maduración de los estilos y los estigmas, desarrollándose con menor precocidad las papilas estigmáticas. Es necesario tener en cuenta para posteriores estudios basados en la reproducción de la especie los descriptores propuestos en el presente trabajo para estimar la incidencia de la asincronía floral.

Por último, se debe prever que al ser Jatropha curcas L. una especie poco domesticada con polinización 
cruzada, su estructura genética es bastante heterogénea y se puede suponer que muchos de sus genes se encuentran en condición de heterocigosis. Desde esta perspectiva, se requieren estudios que permitan establecer el efecto deletétero que la homocigosis de algunos de sus loci pueda causar al obtener líneas endogámicas. La obtención

\section{REFERENCIAS}

Adolf W, Opferkuch HJ, Hecker E. Irritant phorbol derivatives from 4 Jatropha species. Phytochemistry 1984;23 (1): 129 - 132.

Bertin RI. Incidence of monoecy and dichogamy in relation to self-fertilization in angiosperms. American Journal of Botany 1993; 80:537-560.

Campuzano LF. Perspectivas de la investigación de Jatropha curcas L. en Colombia PARTE I: Componente Genético. Revista Facultad Nacional de Agronomía, Medellín. 2009;62 (3):51-63.

Consejo Agropecuario del Sur (CAS). 2009. Red de Coordinación de políticas Agropecuarias (REDPA). Grupo de trabajo sobre Políticas públicas en Agroenergía -GT 6:p. 15- 18.

Downes RDy Wold C. Biodiversity prospecting rules of the game. BioScience. 1994; 44(2): 95-98.

Fox JL. Biodiversity promises great prospecting. Bio / Technology. 1995; 13(6):544-545.

Gohil RH, Pandya JB. Genetic Diversity Assessment in physic nut (Jatropha curcas L.). Central Salt and Chemical Research Institute (SCRI). Journal Bhavnagar India. 2008;2(4):321-326.

González F, Fita JM. 2001. Conservación y caracterización de recursos fitogenéticos. Madrid: INIA, $279 p$.

Guerrero JA. 2010. Tesis “Caracterización Morfológica yAgronómica de la Colección Nacional de Germoplasma del Piñón (Jatropha curcas L.), de Corpoica-La Libertad. Universidad de los Llanos (Unillanos). Villavicencio, Colombia. $150 \mathrm{p}$.

Heller, J. 1996. Physic nut; Jatropha curcas L. IPGRI IPK Italy Germany (c) International Plant Genetic Resources Institute, p. 24-29. de líneas endocriadas es absolutamente necesaria en programas de mejoramiento tradicional para formar un banco de progenitores a partir del cual se puedan obtener generaciones segregantes que exploten la variación genética de la colección sobre la cual realizar procesos de selección que permitan desarrollar óptimamente el potencial de esta especie para la producción de biodiesel.

Kearns CA, Inouye DW. 1993. Techniques for pollination biologists, University Press of Colorado, Niwot, Colorado. 80544, U.S.A, p.112- 115.

Laviola, G. Bruno, Bhering, L. Leonardo, Mendoncal Simone, Rosado, B. Tatiana y Albrecht, Julio Cesar. Caracterização Morfo-Agronômica do Banco de Germoplasma de Pinhao Manso na Fase Jovem. EMBRAPA. Biosci J Uberlândia. 2011;27 (3):371-379.

Manco E, Pérez D. 2009. Implementación de un Banco de Germoplasma de ecotipos de Piñón en Perú. Ministerio deAgricultura. Instituto Nacional de Innovación Agraria. San Martín, Perú. 46 p.

Martin G, Mayeux A. Reflexiones sobre cultivos oleaginosos energéticos. "II Piñón de la India (Jatropha curcas L.). Un carburante posible". Oleagineux. 1984; 39 (5):283-287.

Mishra D. Selection of candidate plus phenotypes of Jatropha curcas L. using method of paired comparisons. Biomass and Bioenergy 2009; 33:542-545.

Nei M, Li WH. 1979. Mathematical model for studying genetic. Variation in terms of restriction endonucleases. Proceedings of the National Academy of Sciences of the United States of America. 76(10):5269-5273.

Panigrahi S, Francis BJ, Cano LA, Burbage MB. Toxicity of Jatropha curcas seeds from Mexico to rats and mice. Nutr Rep Int. 1984;29(5):1089-1099.

Pedraza EA, Cayón DG. Caracterización morfofisiológica de Jatropha curcas L. variedad Brasil cultivada en dos zonas de Colombia. Universidad Nacional de Colombia. ActaAgronomica. 2010;59 (1): 30-36.

Toral O. Jatropha curcas L., una especie arbórea con potencial energético en Cuba. Pastos y Forrajes. 2008; 31 (3): 191. 